\title{
Flowcytometric Analysis of Lymphocytapheresis in a Patient with Recurrent FSGS after Renal Transplant
}

\author{
Kazuhiro Okano ${ }^{1}$, Himiko Sugimoto ${ }^{1}$, Hikohiro Jinnai ${ }^{2}$, Tomihito Iwasaki ${ }^{2}$, Mari Takano ${ }^{1}$, \\ Misao Tsukada ${ }^{1}$, Naoko Miwa ${ }^{2}$, Naoki Kimata ${ }^{2}$, Kosaku Nitta ${ }^{1}$ and Takashi Akiba ${ }^{2}$
}

\begin{abstract}
Frequently, focal segmental glomerulosclerosis (FSGS) recurs after renal transplantation, resulting in poor graft survival. Pathological mechanisms of the recurrence are still unknown, but both B and T cell disorders are suspected based on much evidence. This supports theoretical benefits using plasma exchange (PE) and lymphocytapheresis (LCAP). A renal transplant was performed for a 35-year-old woman, who suffered steroid-resistant FSGS and developed to chronic kidney disease stage 5D at 31 years old. We treated the patient with recurrent FSGS by LACP and examined whether peripheral neutrophils were dynamically changed after the therapy. Further, we performed flowcytometric analysis to examine lymphocyte fractions before and after LCAP. The decrease of helper (CD4 positive) and memory (CD4 and CD45RO positive) T cells was prominent after LCAP. Although B cells were at the nadir because of rituximab treatment, LCAP also decreased peripheral B cells. These suggest that LCAP has the potential to suppress the activities of recurrent FSGS after renal transplant.
\end{abstract}

Key words: apheresis, focal glomeruloscrelosis, renal transplant

(Intern Med 50: 3009-3012, 2011)

(DOI: 10.2169/internalmedicine.50.6088)

\section{Introduction}

Reccurent focal segmental glomerulosclerosis (FSGS) after renal transplantation is frequently observed: it is associated with poor graft survival (1). There are two clinical presentations of FSGS after transplantation: an early recurrence, the most frequent, characterized by a massive proteinuria within hours to days after implantation of the new kidney and a late recurrence which develops insidiously several months or years after transplantation (2). Basically, pathophysiology of the disease is unknown, but secretion of a circulating factor is suspected to play a key role of inexcessive glomerular permeability. A circulating factor bound to protein A or a complex with immunoglobulins is reported as possible pathogenic factor $(3,4)$. A $\mathrm{T}$ cell disorder is suspected based on much evidence for Th2 cytokine bias in recurrent FSGS. Complementary DNA library from $\mathrm{T}$ cells of patients with relapsing FSGS showed transactivated inter- leukin (IL)-4 gene and down-regulated interferon- $\gamma$ expression (5). These data theoretically support that plasma exchange (PE) and lymphocytapheresis (LCAP) can markedly reduce urinary protein excretion and sometimes induce complete remission in some cases, but they usually fail to achieve sustained remission (6). It is reported that neither total number of peripheral white blood cells nor the CD4/ CD8 ratio change but that the total number of lymphocytes, $\mathrm{T}$ cells, B cells, and HLA-DR-positive activated $\mathrm{T}$ cells decrease significantly in the response group (7). However, the changes are frequently different among the patients with or without responses to the therapy. There is still no consensus about the timing and frequency of LCAP. LCAP is frequently performed to treat ulcerative colitis, suggesting that effective removal of the activated leukocytes and the retention of naïve leukocytes in the circulation are achieved by LCAP (8). To examine how LCAP affect peripheral blood leukocytes in recurrent FSGS, we performed LCAP session with 6 liters of blood processing and examine fractions of

${ }^{1}$ The 4th Department of Medicine, Tokyo Women's Medical University, Japan and ${ }^{2}$ Department of Blood Purification, Tokyo Women's Medical University, Japan

Received for publication June 30, 2011; Accepted for publication September 11, 2011

Correspondence to Dr. Kazuhiro Okano, kaokano@kc.twmu.ac.jp 


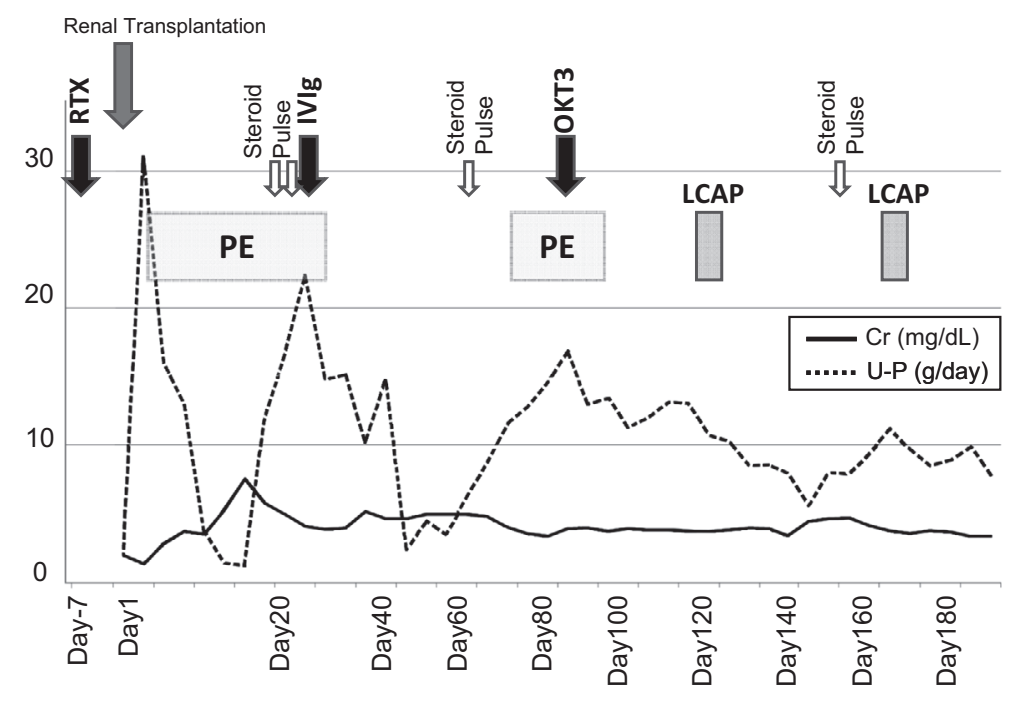

Figure 1. Clinical course of this case. Plasma exchange (PE) combined with several immunosuppressive therapies failed to induce continuous remission of recurrent FSGS after renal transplantation. Cr: serum creatine levels, U-P: proteinuria, IVIg: intravenous immunoglobulin infusion, LCAP: lymphocytapheresis, RTX: rituximab

Table 1. Fractional Analysis of Peripheral White Blood Cells before, during, and after LCAP

\begin{tabular}{|c|c|c|c|}
\cline { 2 - 4 } \multicolumn{1}{c|}{} & $\begin{array}{c}\text { Before } \\
\text { LCAP }\end{array}$ & $\begin{array}{c}\text { 3 Liters } \\
\text { Treatment }\end{array}$ & $\begin{array}{c}\text { After } \\
\text { LCAP }\end{array}$ \\
\hline $\begin{array}{c}\text { Granulocyte } \\
\text { (Neutrophile) }\end{array}$ & $\begin{array}{c}1339 / \mathrm{mm}^{3} \\
(52.1 \%)\end{array}$ & $\begin{array}{c}212 / \mathrm{mm}^{3} \\
(28.0 \%)\end{array}$ & $\begin{array}{c}2718 / \mathrm{mm}^{3} \\
(75.5 \%)\end{array}$ \\
\hline \hline Monocyte & $\begin{array}{c}210 / \mathrm{mm}^{3} \\
(8.2 \%)\end{array}$ & $\begin{array}{c}19 / \mathrm{mm}^{3} \\
(2.5 \%)\end{array}$ & $\begin{array}{c}198 / \mathrm{mm}^{3} \\
(5.5 \%)\end{array}$ \\
\hline \hline Lymphocyte & $\begin{array}{c}968 / \mathrm{mm}^{3} \\
(37.7 \%)\end{array}$ & $\begin{array}{c}528 / \mathrm{mm}^{3} \\
(69.5 \%)\end{array}$ & $\begin{array}{c}684 / \mathrm{mm}^{3} \\
(19.0 \%)\end{array}$ \\
\hline
\end{tabular}

peripheral blood leukocytes using flowcytometric analysis.

\section{Case Report}

A 35-year-old woman visited our hospital in June 2010 for purpose of renal transplantation. At the age of twentytwo, she was diagnosed as FSGS by renal biopsy. FSGS was steroid resistant and relapsed frequently so that lowdensity lipoprotein (LDL) apheresis was performed several times. However, renal dysfunction progressed gradually and she was introduced to hemodialysis (HD) therapy in July 2006. She had almost no native urine output at the time she received a kidney transplant from her mother that was one HLA mismatch. No additional abnormalities were noted in the laboratory data, other than those indicative of end-stage chronic kidney disease (CKD).

Three sessions of PE were performed just prior to the transplantation, because our immunosuppressive protocol includes PE to remove soluble factors in the serum for recipients with FSGS. Her immunosuppressive therapy started 7 days before transplantation with 2 g/day of mycophenolate mofetil, $4 \mathrm{mg} /$ day of tacrolimus, $200 \mathrm{mg}$ intravenous infusion of rituximab (RTX), and $20 \mathrm{mg} /$ day of methylprednisolone. The recipient received a living-donor blood-typematched renal allograft from her mother, placed in the right iliac fossa, on June 15, 2010. Renal biopsy during operation showed only moderate arteriosclerosis in the transplanted kidney. Postoperatively, the allograft functioned promptly. Within several days proteinuria increased above $30 \mathrm{~g}$ per day (Fig. 1). On Day 20 after operation, a biopsy was performed and it showed recurrent FSGS accompanied with wide-ranging podocyte foot process effacement and collapse of capillary lumens. The patient underwent pulse therapy of corticosteroid (500 mg of hydrocortisone for 3 days), infusion of immunoglobulin (IVIg), and PE therapy several times. At glance, PE and IVIg were thought effective to decrease the activities of recurrent FSGS. However, the activities increased again on day 70 and the patient underwent further immunosuppressive therapies including infusion of anti-CD3 antibodies (OKT3). But proteinuria levels moved between 10 to $15 \mathrm{~g}$ per day, while serum creatinine levels were relatively stable at $4 \mathrm{mg} / \mathrm{dL}$.

LCAP was employed to decrease the activity of recurrent FSGS further. The patient was treated with Cellsorba (Asahi Medical Co., Osaka, Japan). A volume of $3,000 \mathrm{~mL}$ of whole blood were processed with a blood flow of $50 \mathrm{~mL} /$ $\min$ in the first two sessions at day 134 and 137, and 6,000 $\mathrm{mL}$ of whole blood in the last two sessions at day 159 and 162. Nafamostat mesilate was used as anticoagulant. No adverse effect of LCAP was observed throughout the course of this therapy. We performed immunological examination including flowcytometric analysis before, during, and after LCAP on day 162. Table 1 shows the dynamics of peripheral blood leukocytes before, during, and after LCAP. A marked reduction was observed in all three types of leukocytes after 3 liters of treatment but an over-shooting increase 


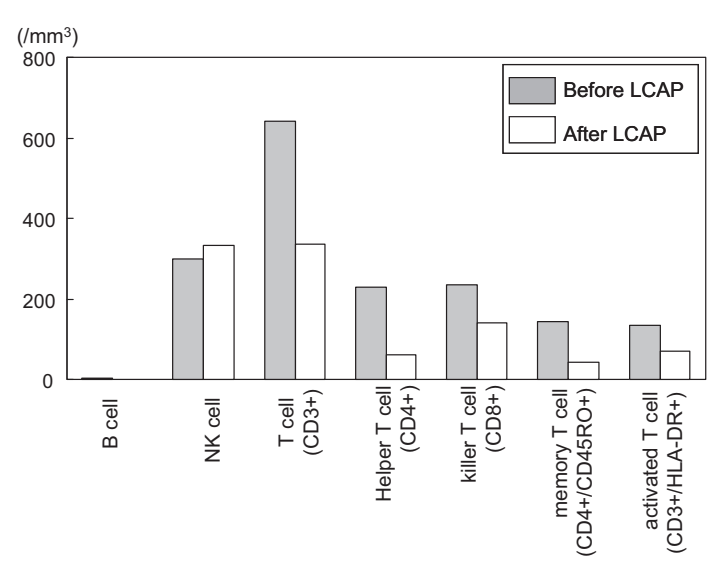

Figure 2. Flowcytometric analysis of peripheral lymphocytes before and after LCAP. Blood samples were collected just before and after the treatment. After erythrocytes were lyzed, whole blood cells were treated with monoclonal antibodies against CD3, CD4, CD8, CD20, CD45, and HLA-DR obtained from BD Biosciences (San Jose, CA, USA). These antibodies were directly conjugated with either fluoresceinsothiocyanate or phycoerythrin. Double immunostaining for CD3/CD4, CD3/CD8, CD4/CD8, CD4/CD45RO, and CD4/ HLA-DR was then performed. All flow cytometric analyses were performed with a FACSscan (Beckton Dickinson, Mountain View, CA, USA).

of neutrophils was observed after the treatment. Although the over-shooting phenomenon was observed in granulocytes and monocytes, the 6-liter LCAP treatment was still effective to reduce peripheral lymphocytes. Flowcytometric analysis was performed to examine fractional dynamics of lymphocytes before and after 6-liter LCAP treatment (Fig. 2). All of the fractions of lymphocytes except natural killer cells (NK cells) were decreased by the treatment. In particular, the decrease of helper (CD4 positive) and memory (CD4 and CD45RO positive) $\mathrm{T}$ cells was prominent. The number of peripheral B cells was already at the nadir because of RTX treatment but showed $75 \%$ reduction after the treatment. These findings suggest that LCAP is effective to efficiently reduce $\mathrm{T}$ cells, especially CD4 positive $\mathrm{T}$ cells.

\section{Discussion}

Recurrence is a major risk in renal transplantation for patients with FSGS. Hariharan et al documented recurrence in at least $53 \%$ of their patients with allograft FSGS (9). Despite the introduction of new immunosuppressive regimens, the incidence of FSGS recurrence has remained unchanged (10). RTX was introduced for decreasing the recurrence of FSGS after transplantation (11). RTX seems to induce remission in about $50 \%$ of cases, but there is still controversy regarding the timing and dose of infusion of RTX (12). Furthermore, there is no data on the long-term side effects with this treatment. The case reported here showed short-period effectiveness of RTX but no continuous effect of RTX was observed. In this case, OKT3 was also used and showed a slight effect to suppress proteinuria, resulting in partial remission of recurrent FSGS. However, many reports suggest that remission of the disease may not be apparent for up to nine months after OKT3 therapy, necessitating extended follow-up to determine responsiveness (13).

Recurrence of FSGS can develop any time within the first two years after renal transplant. Sometimes proteinuria is developing within a few hours post renal transplant, suggesting the presence of a circulating glomerular permeability factor. $\mathrm{PE}$ is the most beneficial when used early in the course of recurrence (14). Protein A immunoadsorptive therapy decreases proteinuria by about $80 \%$ in recurrent FSGS, but the therapy is mostly reported in combination with PE $(4,15)$. In the present case, PE was frequently performed early in the recurrence accompanied with steroid pulse therapy, IVIg, or OKT3. These combined therapies were effective to decrease proteinuria for a few weeks.

The independent effect of LCAP is difficult to examine in the present case. This is a limitation of this case report. However, LCAP therapy is primarily performed in recurrent FSGS, which exhibits resistance against many conventional immuno-suppressive therapies. The precise mechanisms of the therapeutic effect of LCAP are still unclear. LCAP is reported to reduce the number of lymphocytes, resulting in decrease of circulating immunopathgenic factors from peripheral blood cells (16). Consistent with the previous report, the serum levels of immunoglobulins, complements, or lipids changed very slightly before and after the LCAP treatment (17). Removal of activated lymphocytes and induction of naïve lymphocytes may be the most prominent effect of LCAP. RTX or OKT3 is not enough to suppress proteinuria in recurrent FSGS, suggesting that both $\mathrm{B}$ and $\mathrm{T}$ lymphocytes contribute recurrence of FSGS. Therefore, concurrent removal of activated $\mathrm{B}$ and $\mathrm{T}$ lymphocytes by LCAP is expected useful to decrease the activities of recurrent FSGS. In contrast to the case reported by Shimizu et al (18), the present case had been treated with RTX before transplantation and peripheral B cells were kept at the nadir during observation. Another difference was that lymphocyte subsets were changed by LCAP. At present, we think that the decrease in the total number of lymphocytes is not necessarily associated with the therapeutic effect of LCAP therapy in recurrent FSGS.

As far as we reviewed, the report by Rooney et al is the only one to examine the dynamics of immunological status other than our case. CD4/8 ratio of circulating $\mathrm{T}$ cells decreased with LCAP therapy in a rheumatoid arthritis patient (19). This is consistent with the present result even though LCAP removes lymphocytes with nonselective manner. Predominant removal of CD4 T cells may be due to lymphocyte kinetics of the disease background and concomitant drug therapy. Another advantage of LCAP is that the treatment does not require a blood product unlike PE. In conclusion, LCAP has the potential to suppress the activities of recurrent FSGS after renal transplant. In the present case, 
LCAP decreased CD4 positive cells, and in paticular helper and memory $\mathrm{T}$ cells were more prominently decreased than other fractions of peripheral blood lymphcytes.

The authors state that they have no Conflict of Interest (COI).

\section{References}

1. Pardon A, Audard V, Caillard S, et al. Risk factors and outcome of focal and segmental glomerulosclerosis recurrence in adult renal transplant recipients. Nephrol Dial Transplant 21: 1053-1059, 2006.

2. Ponticelli C. Recurrence of focal glomerular sclerosis (FSGS) after renal transplantation. Nephrol Dial Transplant 25: 25-31, 2010.

3. Savin VJ, Shama R, Shama M, et al. Circulating factor associated with increased glomerular permeability to albumin in recurrent focal segmental glomerulosclerosis. N Eng J Med 334: 878-883, 1996.

4. Dantal J, Bigot E, Bogers W, et al. Effect of plasma protein adsorption on protein excretion in kidney-transplant recipients with recurrent nephritic syndrome. N Eng J Med 330: 7-14, 1994.

5. Grimbert P, Valanciute A, Audard V, et al. Truncation of C-mip (Tc-mip), a new proximal signaling protein, induces c-maf Th2 transcription factor and cytoskeleton reorganization. J Exp Med 198: 797-807, 2003.

6. Artero M, Biava C, Amend W, Tomlanovich S, Vincenti F. Recurrent focal glomerulosclerosis: natural history and response to therapy. Am J Med 92: 375-383, 1992.

7. Yokoyama H, Wada T, Zhang W, Yamaya H, Asaka M. Advances in apheresis therapy for glomerular diseases. Clin Exp Nephrol 11: 122-127, 2007.

8. Yamaji K, Yang K, Tsuda H, et al. Fluctuations in the peripheral blood leukocytes and platelet counts in leukocytapheresis in healthy volunteers. Ther Apher 6: 402-412, 2002.

9. Hariharan S, Adams MB, Brennan DC, et al. Recurrent and de novo glomerular disease after renal transplantation: a report from
Renal Allograft Disease Registry (RADR). Transplantation 68: 635-641, 1999.

10. Hubsch H, Montane B, Abitbol C, et al. Recurrent focal glomerulosclerosis in pediatric renal allografts: the Maimi experience. Pediatric Nephrol 20: 210-216, 2005.

11. Hristea D, Hadaya K, Marangon N, Buhler L, Villard J, Morel P, Martin PY. Successful treatment of recurrent focal segmental glomerulosclerosis after kidney transplantation by plasmapheresis and rituximab. Transplant Int 20: 102-105, 2007.

12. Yabu JM, Ho B, Scandling JD, Vincenti F. Rituximab failed to improve nephritic syndrome in renal transplant patients with recurrent focal segmental glomerulosclerosis. Am J Transplant 8: 222227, 2008.

13. Hickson LJ, Gera M, Amer H, et al. Kidney transplantation for primary focal segmental glomerulosclerosis: Outcomes and response to therapy for recurrence. Transplantation 87: 1232-1239, 2009.

14. Vinai M, Waber P, Seikaly MG. Recurrence of focal segmental glomerulosclerosis in renal allograft: an in-depth review. Pediatr Transplant 14: 314-325, 2010.

15. Dantal J, Testa A, Bigot E, Soulillou JP. Effect of plasma protein A immunoadsorption on idiopathic nephrotic syndrome recurring after renal transplantation. Ann Med Interne 143 (Suppl 1): 48-51, 1992.

16. Andoh A, Ogawa A, Kitamura K, et al. Suppression of interleukin-1 beta- and tumor necrosis factor-alpha-induced inflammatory responses by leukocytapheresis therapy in patients with ulcerative colitis. J Gastroenterol 39: 1150-1157, 2004.

17. Yokoyama H, Shimizu M, Wada T, et al. The beneficial effects of lymphocytapheresis for treatment of nephrotic syndrome. Ther Apher 6: 167-173, 2002.

18. Shimizu M, Kitagawa K, Nishio S, et al. Successful treatment of recurrent focal segmental glomerulonephritis after renal transplantation by lymphocytapheresis and rituximab. Transpl Int 23: e53e55, 2010.

19. Rooney M, Whelan A, Feighery C, et al. Changes in lymphocyte infiltration of the synovial membrane and the clinical course of rheumatoid arthritis. Arthritis Rheum 32: 361-369, 1989.

(C) 2011 The Japanese Society of Internal Medicine http://www.naika.or.jp/imindex.html 